\title{
Dosing Common Medications in Hospitalized Pediatric Patients with Obesity: A Review
}

\author{
Barbara Ameer \\ Rutgers University \\ Michael Weintraub \\ Thomas Jefferson University
}

Follow this and additional works at: https://jdc.jefferson.edu/medfp

Part of the Pediatrics Commons

Let us know how access to this document benefits you

\section{Recommended Citation}

Ameer, Barbara and Weintraub, Michael, "Dosing Common Medications in Hospitalized Pediatric Patients with Obesity: A Review" (2020). Department of Medicine Faculty Papers. Paper 267. https://jdc.jefferson.edu/medfp/267

This Article is brought to you for free and open access by the Jefferson Digital Commons. The Jefferson Digital Commons is a service of Thomas Jefferson University's Center for Teaching and Learning (CTL). The Commons is a showcase for Jefferson books and journals, peer-reviewed scholarly publications, unique historical collections from the University archives, and teaching tools. The Jefferson Digital Commons allows researchers and interested readers anywhere in the world to learn about and keep up to date with Jefferson scholarship. This article has been accepted for inclusion in Department of Medicine Faculty Papers by an authorized administrator of the Jefferson Digital Commons. For more information, please contact: JeffersonDigitalCommons@jefferson.edu. 
Title: Dosing Common Medications in Hospitalized Pediatric Patients with Obesity: A Review

\author{
Authors and Affiliations: \\ Barbara Ameer, Department of Medicine, Rutgers - Robert Wood Johnson Medical \\ School, New Brunswick NJ, USA 08854 \\ Michael A. Weintraub, Department of Medicine, Thomas Jefferson University \\ Hospital, Philadelphia PA 19107
}

Keywords: Childhood Obesity, Adolescents, Pharmacologic Therapy, Drug

Treatment, Clinical Studies

Running Title: Common Drugs Studied in Pediatric Patients with Obesity

Contact Information: Dr. Weintraub, Department of Medicine, Thomas Jefferson

University Hospital, 1334 Walnut Street, Suite 602, Philadelphia PA, USA 19107

michael.weintraub@jefferson.edu

Word count: Main text: 5,991 Abstract: 199

Figures and tables: 4 ( 3 tables [ 2 supplemental], 1 figure)

References: 94

Funding: No funding was received in support of this work.

Disclosure: The authors declared no conflict of interest.

Views expressed are the authors, not an official position of their institutions.

Preliminary findings of this work were presented at the American College of Clinical

Pharmacology Annual Meeting, North Bethesda MD, USA, 23-25 Sep 2018. 


\section{Study importance questions}

\section{What major reviews have been published}

- In a major review in JAMA Pediatrics (Harskamp-van Ginkel et al., 2015), coverage was exclusively drug disposition studies published through 2012 , yielding mostly uncommon drugs with pediatric data. Another pediatric paper (Kyler et al., 2019) reviewed 23 drugs frequently prescribed to pediatric inpatients.

- Other journal reviews were similarly limited in scope to pharmacokinetics and/or one therapeutic category, i.e., antibiotics, or clinical setting, i.e., surgery or emergency medicine.

\section{What this review adds}

- Our up-to-date literature review provides a critical appraisal of pediatric obesity clinical research, covering 70 medications in common use among hospitalized pediatric patients in the U.S.

- This overview identifies trends and knowledge gaps which will help prioritize research direction to focus on areas of greatest clinical need. 


\section{Abstract}

Medication management in children and adolescents with obesity is challenging since both developmental and pathophysiologic changes may impact drug disposition and response. Evidence to date indicates an effect of obesity on drug disposition for certain drugs used in this population. This work identified published studies evaluating drug dosing, pharmacokinetics, and effect in pediatric patients with obesity, focusing on 70 common medications used in a pediatric network of 42 U.S. medical centers. A PubMed search revealed 33 studies providing pharmacokinetic and/or effectiveness data for $23 \%$ (16/70) of medications, $44 \%$ of which have just one study and can be considered exploratory. This work appraising 4 decades of literature shows several promising approaches: greater use of pharmacokinetic models applied to prospective clinical studies, dosing recommendations derived from both pharmacokinetics and safety, and multi-year effectiveness data on drugs for chronic conditions (e.g., asthma). Most studies make dose recommendations, but are weakened by retrospective study design, small study populations, and no controls or historic controls. Dosing decisions continue to rely on extrapolating knowledge, including targeting systemic drug exposure typically achieved in adults. Optimal weight-based dosing strategies vary by drug and warrant prospective, controlled studies incorporating pharmacokinetics and modeling and simulation to complement clinical assessment. 


\section{Introduction}

Obesity is becoming a common, chronic metabolic disease, with BMI and obesity rates rising dramatically in recent decades (1). This pattern is evident among pediatric populations in the United States as well as in overweight and obesity trends internationally $(2,3)$. Among those residing in countries with developing economies, $23 \%$ are in the overweight or obese category, according to country definitions of these terms relative to the reference population (4). Currently $18.5 \%$ of U.S. children and adolescents have obesity and $5.6 \%$ have severe obesity $\left(\mathrm{BMI} \geq 120 \%\right.$ of the $95^{\text {th }}$ percentile of BMI-for-age, or an absolute $\mathrm{BMI} \geq 35 \mathrm{~kg} / \mathrm{m}^{2}$, according to the $\left.\mathrm{CDC}\right)(5,6)$.

Obesity is a progressive disease associated with pathophysiological changes in multiple organ systems: respiratory, hepatic, renal, cardiometabolic (hypertension, atherosclerosis, dyslipidemia) and insulin resistance, even among children with metabolically healthy obesity $(7,8)$. When obesity continues into adulthood, there are long-term health complications, e.g., cardiovascular events, especially for individuals with severe obesity (9).

Children with obesity may be prescribed medication at a higher rate than children without obesity, as suggested by self-reported medication use in a longitudinal cohort study in the UK (10). A similar association was found among adolescents in a Canadian epidemiology study (11). Indications may include the management of obesity 
comorbidities, most notably respiratory conditions, as well as perioperative care for orthopedic procedures, adenotonsillectomy, cholecystectomy and metabolic surgery in older children and adolescents. Solmi et al (10) recognized that their study was underpowered to detect significant differences in usage of specific therapeutic agents. Grouping medications by therapeutic classification, they found a strong association for high usage in children with obesity of medications for endocrine, respiratory and central nervous system conditions.

Despite the clinical need for medication, obesity has not been routinely examined as a subpopulation within pediatrics in most clinical drug development programs, as determined by a recent comprehensive review of FDA databases and drug labels (12). Consequently, there is insufficient evidence-based dosing guidance for prescribing drugs in children with overweight or obesity (13).

A systematic review of drug dosing in pediatric obesity published in 2015 was limited to studies with pharmacokinetic data (14). A review such as the present work allows a comprehensive scope to reviewing and critiquing published literature on broad issues. The purpose of this review is to evaluate the extent to which published clinical studies on common pediatric medications inform the process of prescribing in children and adolescents with obesity. 


\section{Methods}

\section{Data Sources and Management}

We began by identifying drugs in common use among hospitalized children and adolescents. Data collected in a retrospective cohort study were utilized (15). The Pediatric Health Information System (PHIS), the administrative database of a national pediatric network, was the source for drug exposure data among 54,549 patients cared for in 2011-2012 and discharged from 42 ICUs, excluding neonatal ICUs, of general children's hospitals across the U.S. (15). Data captured as Clinical Transaction Codes were converted to National Drug Codes and recorded by harmonized terminology using standardized dictionary of drug names.

Therapies were ranked by extent of exposure, or prevalence, expressed as a percentage of patients exposed to the drug at any time during a PICU stay. Table S1 displays a ranked list of drugs with exposures of $14 \%$ or greater.

In preparation for the literature review, we identified drugs with a prevalence of $\geq 5 \%$, provided the drug attained this threshold exposure rate in at least 2 of the age categories: infants, children and adolescents. Where appropriate we extended below that threshold to include metformin and to consider related or interchangeable drugs in a category to allow for differences among hospital formularies. We excluded therapeutic agents with prevalence $\geq 5 \%$ if they are not commonly encountered outside of critical care (e.g., dopamine, epinephrine, glycopyrrolate, lidocaine, milrinone) or in the immediate peri-surgical period (e.g., neuromuscular blocking agents, propofol). Also 
excluded were blood products, ocular products, parenteral fluids, electrolytes and parenteral nutrition. With these additions and exclusions, the medication list totaled 70 for searching published literature.

\section{Search Strategy and Study Selection}

English language PubMed articles from January 1970 - November 2018 were searched using the search string shown in Figure 1. Drug names for the search were from the PICU list were also prevalent among general pediatric inpatients (16). The drugs consisted of 70 medications in 8 therapeutic categories: analgesics, antimicrobials, asthma/allergy/inflammation, cardiovascular, central nervous system, coagulation, diabetes and gastroenterology. We excluded reports on drugs used predominately in pediatric anesthesia or procedural sedation.

Authors independently reviewed abstracts of articles identified by the search. For articles identified by this search, reference lists were examined for additional data sources that met our criteria. Additional articles not found in the original search were considered for inclusion. This aspect deviated from the strict criteria of a systematic review approach.

Results were screened for inclusion of pediatric studies or case series of dosing strategies in association with efficacy or effectiveness measures, and/or pharmacokinetics. We included published pediatric studies in which the study population represented a spectrum of body sizes, provided outcome data were 
presented separately for the group with overweight or obesity or where data were combined, the percentage of individuals with overweight or obesity was $\geq 20 \%$. The literature search process is displayed in a flow diagram consistent with the PRISMA statement (17) (Figure 1).

From each study included in this review, we collected patient population size, age, body size categories; drug dosing information; study design; outcomes and key findings that might impact dosing in obesity.

\section{Findings: An Overview}

The PubMed search initially returned 466 records (Figure 1), and most were eliminated after abstract review because they were not relevant. With 6 additional articles from manual searching, final results consisted of 33 studies.

The published literature indicated substantial gaps in knowledge about drug dosing and response in childhood obesity. For 70 medications in common use among hospitalized pediatric patients, only $23 \%$ (16/70) of these drugs were specifically studied in pediatric patients with obesity (Table 1). Key study features extracted from the publications are displayed in Table S2: population, design, dosing, PK and other outcome data, were and. Also, the column "Possible Dosing Impact in Obesity? yes/no" suggests which 
findings, particularly if replicated in additional pediatric studies, would translate to needing dose modification in pediatric patients with obesity.

Among the 33 investigations, many enrolled fewer than 40 pediatric patients.

Exceptions were several retrospective antimicrobial pharmacokinetics studies and two effectiveness studies of asthma medications, one which retrospectively evaluated 103 children with obesity taking inhaled budesonide and another retrospective study of inhaled beta-agonists evaluated 1066 pediatric patients with obesity. Where multiple studies existed on a given drug, heterogeneity precluded pooling for secondary analysis. Of the 16 drugs investigated, 7 drugs had only one study: albuterol, budesonide, cefazolin, fosphenytoin, metformin, tobramycin and warfarin. Findings from single studies can be considered exploratory, requiring confirmation in new clinical research studies, case series and/or registries.

While most work was published in the past decade, some studies involved data collection over several years. As a result, some dosing schemes employed were no longer current at the time of data analysis, relative to product labeling, institutional practice protocols or published clinical practice guidelines.

Of the publications identified by this review, $64 \%$ (21/33) of studies were conducted retrospectively. Analysis of previously obtained patient data was dominant among antibiotic and anticoagulant drug studies. This retrospective study design underscores the pragmatic approach to clinical research in pediatric subpopulations. 
Antimicrobials collectively represented the most common therapeutic class with published data and vancomycin was the leading single drug studied in pediatric obesity. The next most commonly evaluated categories were anticoagulants and analgesics. Fewer investigations are published in the areas of anti-seizure drugs/sedatives, asthma/allergy/inflammation, diabetes and gastroenterology. We found no studies on common cardiovascular agents.

Dosing was observed to be either a fixed dose or a dose based on weight or other measure of body size. Fixed dosing approaches included fixed-tiered by age category, fixed-tiered by weight or BMI category, or by both age and body habitus. In weight-based dosing, options were total body weight (TBW) or an adjusted measure such as lean body weight (LBW) or ideal body weight (IBW) (Table S2). 


\section{Findings and Discussion by Therapeutic Category}

\section{Analgesics}

Acetaminophen. The single most frequently administered medication, acetaminophen was noted in at least half of patients (15). Despite broad utilization since it was first available by prescription in 1952 (18), few investigations studied the impact of obesity on acetaminophen metabolism, disposition, analgesic effect or toxicity in pediatric patients.

The maximum single dose is $1,000 \mathrm{mg}$ while the daily dose per the product label should not exceed $4,000 \mathrm{mg}$ in both adults and children $\geq 50 \mathrm{~kg}$ to avoid hepatotoxicity (19). Intravenous (IV) acetaminophen has a role in managing fever and postoperative pain, in order to minimize or avoid opiates. Hakim et al. (20) recruited 11 adolescent girls with severe obesity and administered a single $1,000 \mathrm{mg}$ IV dose following bariatric surgery. Subsequent serum acetaminophen concentrations, $<30 \mu \mathrm{mol} / \mathrm{L}(4.5 \mu \mathrm{g} / \mathrm{mL})$, were inadequate for analgesia. Investigators advised targeting a concentration $>66 \mu \mathrm{mol} / \mathrm{L}$ $(10 \mu \mathrm{g} / \mathrm{mL})$ within $2 \mathrm{~h}$ of an IV infusion. That level might be achieved through weightbased dose incorporating some adjustment (e.g., allometric scaling), which would require more research to define.

Barshop et al. (21) compared PK parameters in 12 boys with obesity and nonalcoholic fatty liver disease (NAFLD) to 12 age-matched boys without obesity and with normal liver function. Following a single $5 \mathrm{mg} / \mathrm{kg}$ oral dose not exceeding $325 \mathrm{mg}$, the glucuronide metabolite to acetaminophen ratio, was higher among children with obesity. 
This finding, measured at just one timepoint, suggests enhanced drug conjugation in obesity. Single dose PK parameters, however, were similar between groups, providing no indication that dosing of acetaminophen should be different. Further investigation of acetaminophen's metabolic clearance is warranted using multiple dosing in larger numbers of pediatric patients across a broad body size spectrum.

Fentanyl. PK of fentanyl was evaluated in 6 adolescents with severe obesity (BMI>99 tho) who received IV doses based on IBW in bariatric surgery (22). Clearance was higher, but volume of distribution (V) was not markedly different among these adolescents compared to adult historic controls.

Time to attain individualized goal sedation after the start of an infusion was retrospectively evaluated in 18 patients with obesity and 56 patients without obesity (23). The time to goal sedation was highly variable, regardless of body weight, and only weakly associated with the initial infusion rate.

Antimicrobial Agents

Cefazolin. The $\beta$-lactams are the largest of the various antibiotic classes, yet only one has been studied in pediatric obesity. A weight-based IV dose of cefazolin was prospectively evaluated in 5 children with obesity in a PK study in Japan (24). Dosing weight was an average of TBW and IBW. PK parameters did not differ significantly in the children with obesity compared to historic controls, who were children of normal body weight. 
Clindamycin. Grouped as one of the 32 understudied drugs lacking pediatric dosing advice and safety data, clindamycin was prospectively evaluated for effect of body habitus and age on dosing. As part of the Pharmacokinetics of Understudied Drugs Administered to Children Per Standard of Care trial program (25), PK data were combined with two other prospective studies (26) to develop a population pharmacokinetic (PopPK) model (27). Collectively, the publication encompassed 76 children with obesity and 49 children with healthy body weight, who received clindamycin 20 to $40 \mathrm{mg} / \mathrm{kg}$ based on TBW, up to a maximum daily dose of 2,700 mg, which is at the upper end of the dosing range for adults. The PopPK model confirmed the validity of the practice of dosing clindamycin based on TBW, according to agebased dosing recommendations.

Aminoglycosides. Tobramycin and gentamicin are water soluble molecules known to distribute extensively into extracellular body water. Body fluid dynamics are altered with obesity and developmental age. Some water resides in adipose tissue, particularly extracellular fluid which increases with increasing weight. Consequently, changes in pharmacokinetics of drugs like aminoglycosides which distribute into extracellular body water are more evident in persons with severe obesity (28).

For drugs with a narrow therapeutic index that are known to be highly water soluble, current dosing practice in obesity, in adults and children, is based on IBW plus a percentage of the excess body weight into which the water-soluble drug distributes. In the case of aminoglycosides, the adjusted dosing weight is equal to IBW $+0.4^{*}$ (TBW IBW), where 0.4 is a correction factor correlating with $40 \%$ of excess body weight (29). 
The correction factor may vary for other drugs, according to available knowledge about their partitioning and distribution. IBW is estimated by one of several available equations that utilize readily measurable patient characteristics, i.e., height and sex.

Koshida et al. (24) administered one dose of IV tobramycin to the same Japanese children as in the cefazolin study utilizing an adjusted dosing weight, i.e., an average of TBW and IBW, in recognition of the distribution characteristics of the aminoglycoside. As expected, the predicted volume of distribution at steady state (Vss), expressed as liters per $\mathrm{kg}$ of TBW $(\mathrm{L} / \mathrm{kg})$, was smaller in children with obesity, whereas clearance was similar to pediatric historic controls.

Choi et al. (30) evaluated gentamicin concentrations previously measured in 25 children with obesity and compared the values to control patients of healthy body weight, matched for age, sex and antibiotic indication. Although dosing was based on TBW, the individual dose was capped at $120 \mathrm{mg}$. Gentamicin Vss (L/kg of TBW) was smaller in the children with obesity, consistent with the tobramycin study. Peak concentrations were higher, and concentrations at the end of the $8 \mathrm{~h}$ dosing interval exceeded the advised limit of $4.2 \mu \mathrm{mol} / \mathrm{L}(2 \mathrm{mg} / \mathrm{L})$ in $16 \%$ of patients with obesity but in no children of healthy body weight. The investigators noted that dosing is challenging due to interpatient variability in the amount of extracellular water contained in adipose tissue and the changing composition of body water and fat over the course of normal child development. 
Options for dosing weight and measures of body composition to optimize attainment of therapeutic serum levels continue to be explored. Using modeling and simulation with gentamicin serum concentrations from over 500 pediatric patients across the body weight spectrum, Moffett et al. (31) predicted that an optimized body weight adjustment could be achieved using fat-free mass, as an alternative to IBW. Fat-free mass, which consists of bone, muscles, non-adipose components of organs, extracellular water and blood volume, approximates LBW minus lipid membranes (32). Fat-free mass can be estimated in the clinical environment using readily accessible patient measures, e.g. height and weight (33).

Vancomycin. As one of the oldest approved prescription drugs, introduced in the U.S. in 1952, vancomycin is a mainstay treatment of life-threatening methicillin-resistant $S$. aureus infections.

In adults, changes in $\mathrm{V}$ with excess body weight are inconsistent among published reports (29) whereas clearance increases as body weight increases. Both $V$ and clearance are correlated with TBW rather than LBW in adults with severe obesity (34). TBW is therefore used for initial empiric dosing of vancomycin in adults (35). Dosing intervals may require shortening in patients with obesity and normal renal function to ensure serum vancomycin concentrations remain above the minimum inhibitory concentration (MIC) (36). 
Therapeutic drug monitoring is common in patients with obesity to maintain drug concentrations within a defined range to ensure efficacy. Monitoring has been reported since the 1980s, including exploration of patient-level covariates for vancomycin levels i.e., age, creatinine clearance and body weight.

A total of 8 studies, 7 retrospective and 1 prospective, published from $2011-2015$ and involving over 300 pediatric patients with obesity, characterized the pharmacokinetics of vancomycin and/or described dosing practices in this pediatric subpopulation. By utilizing data from patients who were prescribed this antibiotic by their treating physicians according to institutional standards of care, the investigators took advantage of blood draws for serum concentration measurement, as part of routine medical care.

Two vancomycin reports were PK analyses. In a study of 87 children with overweight or obesity and 87 children of healthy body weight matched for age and renal function, $\mathrm{V}$ was predicted best using TBW. Clearance was best described using TBW to the power of 0.75 , also known as allometric weight (37). In another analysis of 115 children and adolescents over a wide spectrum of body sizes, Camaione and colleagues (38) speculated that defining a desired target range for area-under-the-concentration-time curve (AUC) might be worth exploring as an alternative to target drug concentrations, i.e., peak and trough. They also proposed body surface area (BSA) as an alternative to body weight. Implementing these options may not be feasible in resource-constrained institutions that cannot offer AUC-based vancomycin monitoring services (39). 
The remaining 6 published reports were clinical studies. In the only prospective observational study, dosing vancomycin using a twice daily frequency resulted in vancomycin AUC to MIC ratios and trough concentrations considered clinically inadequate (mean trough, $2.3 \mu \mathrm{mol} / \mathrm{L}[3.4 \mathrm{mg} / \mathrm{L}]$ ) regardless of body weight $(40)$. In the other reports where the dosing interval was shorter, those with obesity were more likely to have somewhat higher mean trough concentrations compared to peers of healthy body weight. The magnitude of the values depended upon the vancomycin dosage regimen, generally ranging between 40 and $60 \mathrm{mg} / \mathrm{kg}$ daily in 3 or 4 divided doses (41, $42,43,44,45)$. It is noteworthy that when dose and dosing interval were stratified by both age and TBW, the mean trough concentrations were close to goal of $>6.9 \mu \mathrm{mol} / \mathrm{L}$ (10 mg/L), i.e., $9.9 \mu \mathrm{mol} / \mathrm{L}(14.4 \mathrm{mg} / \mathrm{L})$ in pediatric patients with obesity, and $7.2 \mu \mathrm{mol} / \mathrm{L}$ $(10.5 \mathrm{mg} / \mathrm{L})$ in those of healthy body weight (42). Thus, it may be optimal when both TBW and age are factored into vancomycin regimens, with younger children requiring higher body weight-based doses and shorter dosage intervals (42). 


\section{Asthma, Allergy and Inflammation}

Albuterol. About one-quarter of new asthma cases in children with obesity can be ascribed directly to obesity, based on electronic U.S. health data (46). Furthermore, obesity is correlated with worse asthma-related outcomes and decreased bronchodilator responsiveness (47) among children who are Black or Latino . In a case-control, crosssectional analysis of nearly 3,000 patients from 2 retrospective studies (48), $36 \%$ had obesity (BMI $\geq 95^{\text {tho }} \%$ ). In this racial/ethnic minority population, the odds of being unresponsive to albuterol were $24 \%$ greater in individuals with obesity compared to children with lower BMI. The bronchodilator-unresponsive patients with obesity were less likely to have well-controlled asthma, despite increased usage of controller medication (i.e., leukotriene receptor inhibitor, inhaled corticosteroid with long-acting beta-2 agonist).

Budesonide. In addition to being severe and poorly responsive to medications, obesityrelated asthma in children is likely nonallergic (49). Forno and colleagues (50) examined the relationship between $\mathrm{BMI}$ and responsiveness to inhaled glucocorticoids in a posthoc analysis of cases from a 4- to 6-y asthma trial. They found a decreased improvement in lung function with budesonide in children with obesity when compared to participants without obesity. Limited by the dose specified in the study protocol, the analysis could not inform whether higher doses might have been more efficacious in obesity.

Central Nervous System 
Fosphenytoin. Therapeutic drug monitoring is a common practice during antiepileptic therapy with phenytoin or its prodrug, fosphenytoin. Messinger et al. (51) retrospectively collected fosphenytoin dosage information and corresponding phenytoin concentrations in 110 children and adolescents across the body weight spectrum. After a mean IV TBW-based loading dose of $23 \mathrm{mg}$ phenytoin equivalents $/ \mathrm{kg}$, the mean \pm standard deviation plasma concentration at 2 to $4 \mathrm{~h}$ was $89 \pm 27 \mu \mathrm{mol} / \mathrm{L}(22.4 \pm 6.8 \mathrm{mg} / \mathrm{L})$. In multivariable linear regression analysis, dose but not body weight, was a significant contributor to serum phenytoin concentrations. Additional drug therapy for control of seizures was not required more often in patients with high BMI. It was concluded that children with obesity and status epilepticus do not require higher $\mathrm{mg} / \mathrm{kg}$ fosphenytoin IV loading doses.

Midazolam. PK of midazolam was prospectively studied in 19 adolescents with obesity or overweight following a single IV dose of 2 or $3 \mathrm{mg}$ (52). Findings supported a fixed dose since TBW was not predictive of clearance. Across the body weght spectrum of 62 to $150 \mathrm{~kg}$, higher TBW was associated with a larger V, which investigators attributed to excess body weight. A subsequent analysis combined data from these adolescents with data from a multicenter study of 20 adults with $\mathrm{BMI}>40 \mathrm{~kg} / \mathrm{m}^{2}$ who received $5 \mathrm{mg} \mathrm{IV}$ (53). The combined data set provided an even broader body size distribution. Findings indicated that clearance in fact increased with higher TBW, and clearance among adolescents was higher than that in adults (54).

\section{Coagulation and Thrombosis}


Enoxaparin - Prophylactic Dosing. Obesity is an independent risk factor for developing venous thromboembolism (VTE). For VTE prophylaxis in the general pediatric population, the American College of Chest Physician clinical practice guidelines specify dosing subcutaneous enoxaparin $0.5 \mathrm{mg} / \mathrm{kg}$ every 12 hours (55). The upper limit of dosing in pediatrics traditionally has been the adult dose (56), which is either $30 \mathrm{mg}$ every 12 hours or $40 \mathrm{mg}$ daily for enoxaparin.

In a retrospective case series, Lewis and colleagues (57) reviewed hospitalizations of 3 adolescent males with obesity or severe obesity initiated on enoxaparin $40 \mathrm{mg}$ once daily for VTE prophylaxis. In all patients, both upward titration of the dose and shortening of the dosing interval to 12 hours was required to achieve the recommended target activated anti-factor X-activated (anti-Xa) range of 0.1 to 0.3 units $/ \mathrm{mL}$ measured 4 to 6 hours after a subcutaneous injection. Authors speculated that PK differences in adolescents with obesity was due to increased body-fat percentage or higher renal blood flow and increased glomerular filtration rate. Researchers concluded a reasonable approach in obesity would be to start with a fixed dose of a low molecular weight heparin in children $>40 \mathrm{~kg}$ with monitoring of anti-factor Xa levels.

Mushtaq et al. (58) investigated enoxaparin dosing in a prospective study of 4 adolescents with severe obese $\left(\mathrm{BMI}>99^{\text {tho }} \%\right)$ receiving perioperative VTE prophylaxis for bariatric surgery. Individuals with $\mathrm{BMI} \leq 50 \mathrm{~kg} / \mathrm{m}^{2}$ received $40 \mathrm{mg}$ every 12 hours and $\mathrm{BMI}>50 \mathrm{~kg} / \mathrm{m}^{2}$ received $60 \mathrm{mg}$ every 12 hours, which resulted in anti-Xa in the target range, although the levels were lower than historic data from adults of normal BMI. 
Authors concluded this two-tiered, body size-adjusted fixed dosing is adequate for thromboprophylaxis in adolescents with obesity. Further evidence to support that approach as a dosing recommendation was recently provided by a similar prospective study of enoxaparin prophylaxis with fixed doses of either 40 or $60 \mathrm{mg}$ stratified by BMI $<50$ or $\geq 50 \mathrm{~kg} / \mathrm{m}^{2}$, respectively. The BMI-stratified dosing achieved desired anti-Xa activity in 10 of the $12(83 \%)$ adolescent bariatric surgery patients (59).

Enoxaparin - Treatment Dosing. Two pediatric studies examined the effect of dosecapping or reduced doses of enoxaparin for VTE treatment. In a retrospective review of 30 pediatric patients, an average TBW-based dose of $0.8 \mathrm{mg} / \mathrm{kg}$ of enoxaparin was administered to children with obesity, with a maximum of $170 \mathrm{mg}$, compared to 1.1 $\mathrm{mg} / \mathrm{kg}$ in their case-control counterparts (60). Higher initial anti-Xa levels were observed in those with obesity but subsequent anti-Xa levels were in the therapeutic range and early dose adjustment did not appear necessary. Authors concluded no dosing adjustment is necessary in pediatric obesity but closer monitoring over time is warranted to avoid supratherapeutic levels and bleeding events.

Reduced doses ( $\leq 1 \mathrm{mg} / \mathrm{kg} /$ dose every $12 \mathrm{~h}$, the standard initial treatment dose) of enoxaparin were retrospectively examined as a possible approach to avoid supratherapeutic anti-Xa levels in 30 adolescents with overweight or obesity receiving the drug twice daily subcutaneously as treatment for VTE (61). TBW-based initial doses of 0.7 to $\leq 1 \mathrm{mg} / \mathrm{kg}$ were effective, although VTE progressed in 2 adolescents initially dosed below $0.7 \mathrm{mg} / \mathrm{kg}$. During 3 months of treatment, $83 \%$ of anti-Xa levels were in the 
desired range ( 0.5 to 1.0 units $/ \mathrm{mL})$. At the end of treatment, the mean dose, adjusted to target anti-Xa, was $0.8 \mathrm{mg} / \mathrm{kg}$. Bleeding was similar between groups.

Heparin. In a retrospective cohort study of 25 children with obesity, Taylor et al. (62) investigated the effect of weight on coagulation parameters and dosing requirements during a weight-based continuous infusion of heparin. Patients with obesity required significantly lower initial and maintenance infusion rates per kilogram compared to the 25 age- and sex-matched children of healthy body weight. The mean \pm standard deviation infusion rate was $19.1 \pm 6.7 \mathrm{U} / \mathrm{kg} / \mathrm{h}$ in patients with obesity compared to $24.3 \pm$ $9.6 \mathrm{U} / \mathrm{kg} / \mathrm{h}$ in 25 patients without obesity $(p=0.033)$ to achieve stable therapeutic monitoring values. Authors hypothesized this difference was attributable to heparin's less extensive distribution into adipose tissue compared to lean muscle.

In contrast, in another retrospective review examining a single heparin bolus for prophylaxis prior to cardiac catheterization, Moffett et al. (63) found a similar response regardless of body weight with no need to adjust the dose in the 39 pediatric patients with obesity. This cohort study was limited in its use of only a single bolus dose and monitoring with the activated clotting time rather than with a more specific, sensitive monitoring test such as aPTT or anti-Xa activity.

Warfarin. To determine whether obesity affects time to therapeutic INR, Moffett et al. (64) conducted a retrospective cohort evaluation of hospitalized pediatric patients initiated on warfarin therapy, following a standard institutional protocol for initial dosing 
and subsequent titration. The institutional protocol included a maximum initial dose of 5 $\mathrm{mg}$ to prevent overdose and did not accommodate exceptions for the presence of obesity. Time to achieve a therapeutic INR in the 10 patients with obesity was twice as long and more variable compared to 20 age- and sex-matched patients of healthy body weight: median [range] 6 [ 4 to $28 \mathrm{~d}$ ] vs. 3 [ 1 to $10 \mathrm{~d}$ ], $p<0.01$. Authors posit altered warfarin dosing requirements in obesity may be due to increased $\mathrm{V}$ and/or related to elevated triglycerides which correlate with elevated plasma vitamin $\mathrm{K}$ concentrations. Authors suggested that elimination of the dosing cap for patients with obesity may prevent the prolonged time to therapeutic INR observed in this study.

\section{Diabetes}

Metformin. Both metformin and basal insulin are FDA-approved for glycemic management in children and adolescents with type 2 diabetes (T2D) (65). As they age, children with T2D and obesity are likely to have increasingly severe obesity (66). Obesity management is not a labeled indication for metformin, but it is associated with modest weight reduction in children and weight stabilization in adolescents with obesity and insulin resistance $(67,68,69)$.

The effect of overweight and obesity on metformin PK was a pre-specified secondary objective of a randomized, triple-blind, placebo-controlled trial in youth with insulin resistance. The PK sub-study enrolled 17 adolescents with obesity and 5 adolescents with overweight (overall mean TBW, $79 \mathrm{~kg}$; estimated LBW, $49 \mathrm{~kg})(70,71)$. In these 22 outpatients, all of whom had normal renal and hepatic function, metformin clearance 
was higher as weight increased. Transporter genes affecting metformin disposition were evaluated as covariates in the model but did not indicate a contribution from gene polymorphisms.

Oral metformin clearance was higher in that study compared to a study in 4 preadolescent girls who were not obese. It was hypothesized that excess body weight was associated with increased renal tubular secretion through induction of renal transporters (72).

With clearance in children with obesity approaching that reported in adults of healthy body weight, the investigators proposed re-evaluation of the maximum dosage of 2,000 $\mathrm{mg}$ in children with obesity if there is inadequate response. They propose using the maximum daily dose for adults with normal renal function, which is $2,550 \mathrm{mg}$ per the FDA product label (73) and $3000 \mathrm{mg}$, per the Summary of Product Characteristics (74). The current clinical practice recommendation for children is to add insulin to metformin to achieve glycemic control rather than to exceed dosage limits described in the label (66). High doses are often accompanied by fatigue and gastrointestinal adverse effects, which are affected by the rate of up-titration of the metformin dosage. Additionally, children have low tolerability of tablet size and quantity and consequently incomplete medication adherence. These concerns are more prominent in young children (69). In general, tolerability and safety are important considerations when contemplating using maximum adult daily dosages to achieve adult-level systemic drug exposures in children with obesity. 


\section{Gastrointestinal Agents}

Pantoprazole. Proton pump inhibitors (PPIs) are acid suppressive drugs used in the treatment of gastroesophageal reflux disease (GERD), which is common in pediatric patients with obesity. Pantoprazole is the only PPI that has been studied in pediatric obesity. The traditional approach of PPI dosing involves empiric dose escalation for obese individuals given their higher TBW.

Two prospective trials investigated the effect of body weight on pantoprazole PK in children and adolescents $(75,76)$. Individuals with higher BMls exhibited higher systemic pantoprazole exposures and slower drug clearance compared to historic controls (76). Authors cautioned that TBW-based dosing in pediatric patients with obesity would likely generate a higher risk for long-term adverse events (e.g., osteopenia and fractures, mineral and vitamin deficiencies) without additional therapeutic benefit. In contrast, LBW dosing in children and adolescents with obesity achieved similar drug exposure to their counterparts of healthy weight. The study confirmed the variation in PK ascribed to age-related maturation of and genetic polymorphism in CYP2C19, the PPI's primary metabolizing enzyme. Authors concluded dose escalation based on higher weight is not advisable. Dosing can be based on the same guidelines as healthy body weight peers of similar age and drug metabolizing capacity

An extended analysis of data from the original prospective study of 41 children and adolescents with GERD and obesity used simulation in a PopPK model to compare two- 
tiered fixed dosing to LBW- or TBW-based dosing strategy and concluded that fixed dosing strategy is suitable (77). A two-tiered strategy for children $\geq 5 \mathrm{yr}$ is specified in the U.S. product label for delayed release pantoprazole: $20 \mathrm{mg}$ once daily for weight 15 to $<40 \mathrm{~kg} ; 40 \mathrm{mg}$ once daily for weight $\geq 40 \mathrm{~kg}(78)$.

Research is needed to elucidate the mechanism by which obesity affects pantoprazole disposition and to determine whether the dosing approach for pantoprazole can be generalized to other PPIs. 


\section{General Critique and Points of Special Interest}

Our findings indicate that existing published studies on therapeutics in pediatric obesity are sparse and have not focused on some of the most prevalent agents used in hospitalized pediatric patients. When no pediatric information exists in the literature to inform drug use, clinicians and researchers often rely on expert opinion and evidence from adult populations (7). For PK data derived from adult obesity studies, extrapolation to pediatric patients has limitations. With midazolam, for example, findings in an adolescent PK study conflicted with a prior report in adult obesity (54).

A premise for extrapolation is that the disease, its progression and response to intervention are sufficiently similar between adults and children to justify extrapolation for therapeutic decision making (79). It is not established, however, that obesity and its natural history are similar in adult and pediatric populations. Like type 2 diabetes across the age spectrum, there may be distinctions between juvenile onset and adult onset obesity $(80,81)$. Also, the underlying disease being treated may differ between age groups, as is the case for thrombotic disease and obesity-associated asthma in adolescents compared to adults $(49,61)$.

The studies presented here underscore practical challenges in undertaking clinical studies in pediatric subpopulations, i.e., ethics, feasibility and availability of resources to conduct robust clinical research. Utilizing real world data is acknowledged as an 
acceptable pragmatic strategy for pediatric therapeutic research, particularly for PK and PD studies (82).

Most studies identified in this review were conducted retrospectively at a single center, involved small numbers of pediatric patients with obesity, and relied upon administrative databases to capture of information. These aspects can introduce informational bias, bias due to misclassification of obesity status, and selection bias. Combining data from multiple studies, as was done with midazolam for adults and adolescents (54), albuterol for children and adolescents up to the age of 21 y (47) and clindamycin (27), addresses the issue of adequate numbers to help ensure generalizability, however, pooling may introduce additional variability. Given the limitations imposed by study design and study conduct, as described in published drug studies in pediatric obesity, translation of their findings to the clinic should only be done with careful patient monitoring.

Despite their limitations, retrospective studies are useful in generating hypotheses for testing in subsequent prospective trials. An alternate strategy is to prospectively consent parents on behalf of young patients who are, or would soon be, prescribed a medication for biological sample collection on the same schedule as clinical care samples $(27,40,83)$. A third approach is to intensify use of existing clinical research resources designed for adults (84). Specifically, including older adolescents with obesity into clinical studies of adults across a body weight spectrum may be considered. National Institutes of Health clinical research funding in fact now carries the expectation of inclusion of children and adolescents, allowing for exceptions for ethical reasons or 
with scientific justification (85). With the high overall prevalence of overweight and obesity in both genders [23.8\% (22.9 to 24.7$)$ of boys and $22.6 \%$ (21.7 to 23.6$)$ of girls residing in countries with developed economies (4)], it may be feasible to extend clinical research to include this subpopulation in multinational clinical research. The aim is to efficiently acquire knowledge about efficacy or effectiveness, safety and/or drug disposition in children and adolescents in advance of designing and conducting dedicated drug trials in pediatric patients with obesity, if they are considered necessary.

A strength of our review is its broad coverage of 70 common pediatric medications, based on a cross-section of 42 U.S. pediatric medical centers encompassing tens of thousands of patient admissions. We believe this approach to the question of the effect of obesity on drug dosing would identify medications likely to be relevant to general hospital practice. Prior pediatric reviews narrowed their coverage by focusing on one therapeutic category, e.g., antimicrobials (86), area of practice, e.g., surgery (87) or emergency medicine (88), or PK properties of drugs (14). The later major review yielded mostly uncommon drugs. Of the studies we report, over $70 \%$ were published after the completion of their literature review phase in 2012. Another review reported on 23 common inpatient drugs but offered little description and critique of the study designs (89).

A few observations about our work are noted with respect to its scope and process. The range of identified drugs was specifically those in use among U.S. pediatric intensive care patients in 2011 - 2012. While the types of medicine in common use in the acute 
care setting differ only slightly between countries (90), drugs licensed since 2012 are not represented in our literature review. Since then, the armamentarium expanded, but drug utilization patterns tend to be consistent over time as prescribers use drugs with which there is known clinical experience. We evaluated medications prescribed in pediatric hospital practice, which omitted some drugs prescribed more often in settings outside of acute care (91). The inpatient drugs on which our literature search was based did not encompass several drug categories frequently prescribed to US pediatric outpatients. Categories include medications for depression (sertraline, fluoxetine), attention-deficit/hyperactivity disorder (ADHD) and contraception (89, 92). Finally, our literature search was limited to one database, PubMed, albeit over a span of many decades.

\section{Concluding Statement}

Healthcare providers regard obesity as a disease to be taken as seriously as diabetes, heart failure and hypertension (93), yet gaps exist in clinical obesity research for all therapeutic areas of medications in common use in pediatrics. Optimal dosing strategies vary by drug and are not necessarily generalizable even among chemically similar entities. Extrapolation from adult data has limitations.

Despite recent progress in pediatric clinical drug research (94), researchers, drug developers and clinicians should strive to develop high-quality evidence on which to base dosing decisions. Prioritization is warranted for drugs that are extensively used (e.g., acetaminophen), used on a regular daily basis (e.g., medication for ADHD, oral contraceptives), important for management of obesity co-morbidities (e.g., statins, 
respiratory medicines), associated with weight gain during long-term use (e.g., antidepressants and antipsychotics, steroids), expensive (e.g., factor product, recombinant biologics), or characterized by a narrow therapeutic index. 


\section{Table Legends}

Table 1.

Presence of Published Pediatric Obesity Studies of 70 Commonly Used Medications in Children and Adolescents

\section{Supplemental Tables}

Table S1.

Common Drug Exposures (14\% or higher) Among Patients in PICUs at US Children's Hospitals

Table S2.

Common Drugs Evaluated in Children and Adolescents with Obesity: Evidence Tables from 33 Studies 
Common Drugs Studied in Pediatric Patients with Obesity

Figure Title and Caption

Figure 1. PRISMA Diagram

Search string and flowchart of search process, identification and screening of studies for inclusion in the review. PRISMA indicates Preferred Reporting Items for Systematic Reviews and Meta-Analyses. 


\section{References}

1. N.C.D. Risk Factor Collaboration. Worldwide trends in body-mass index, underweight, overweight, and obesity from 1975 to 2016: A pooled analysis of 2416 population-based measurement studies in 128.9 million children, adolescents, and adults. Lancet 2017;390: 2627-2642.

2. Lobstein T, Jackson-Leach R. Planning for the worst: Estimates of obesity and comorbidities in school-age children in 2025. Pediatr Obes 2016;11: 321-325.

3. Cole TJ, Bellizzi MC, Flegal KM, Dietz WH. Establishing a standard definition for child overweight and obesity worldwide: International survey. BMJ 2000;320: 12401243.

4. $\mathrm{Ng} \mathrm{M}$, Fleming $\mathrm{T}$, Robinson $\mathrm{M}$, et al. Global, regional, and national prevalence of overweight and obesity in children and adults during 1980-2013: A systematic analysis for the Global Burden of Disease Study 2013. Lancet 2014;384: 766-781.

5. Hales CM, Fryar CD, Carroll MD, Freedman DS, Ogden CL. Trends in obesity and severe obesity prevalence in US youth and adults by sex and age, 2007-2008 to 2015-2016. JAMA 2018;319: 1723-1725. 
6. Ogden CL, Carroll MD, Lawman HG, et al. Trends in obesity prevalence among children and adolescents in the United States, 1988-1994 through 2013-2014. JAMA 2016;315: 2292-2299.

7. Chidambaran V, Tewari A, Mahmoud M. Anesthetic and pharmacologic considerations in perioperative care of obese children. J Clin Anesth 2018;45: 39-50.

8. Zhao M, Lopez-Bermejo A, Caserta CA, et al. Metabolically healthy obesity and high carotid intima-media thickness in children and adolescents: International childhood vascular structure evaluation consortium. Diabetes Care 2018;42: 119-125.

9. Nathan BM, Moran A. Metabolic complications of obesity in childhood and adolescence: More than just diabetes. Curr Opin Endocrinol Diabetes Obes 2008;15: 21-29.

10. Solmi F, Morris S. Association between childhood obesity and use of regular medications in the uk: Longitudinal cohort study of children aged 5-11 years. BMJ Open 2015;5: e007373.

11. Kuhle S, Fung C, Veugelers PJ. Medication use in normal weight and overweight children in a nationally representative sample of canadian children. Arch Dis Child 2012;97: 842-847. 
12. Vaughns JD, Conklin LS, Long Y, et al. Obesity and pediatric drug development. J Clin Pharmacol 2018;58: 650-661.

13. Gade C, Christensen HR, Dalhoff KP, Holm JC, Holst H. Inconsistencies in dosage practice in children with overweight or obesity: A retrospective cohort study. Pharmacol Res Perspect 2018;6: e00398.

14. Harskamp-van Ginkel MW, Hill KD, Becker KC, et al. Drug dosing and pharmacokinetics in children with obesity: A systematic review. JAMA Pediatr 2015;169: 678-685.

15. Dai D, Feinstein JA, Morrison W, Zuppa AF, Feudtner C. Epidemiology of polypharmacy and potential drug-drug interactions among pediatric patients in ICUs of U.S. children's hospitals. Pediatr Crit Care Med 2016;17: e218-228 [Suppl Appendix 211, Suppl Digital Content 212].

16. Feudtner C, Dai D, Faerber J, Metjian TA, Luan X. Pragmatic estimates of the proportion of pediatric inpatients exposed to specific medications in the USA.

Pharmacoepidemiol Drug Saf 2013;22: 890-898. 
17. Moher D, Liberati A, Tetzlaff J, Altman DG, Group P. Preferred reporting items for systematic reviews and meta-analyses: The PRISMA statement. Ann Intern Med 2009;151: 264-269, W264.

18. Ameer B, Greenblatt DJ. Acetaminophen. Ann Intern Med 1977;87: 202-209.

19. Ofirmev ${ }^{\mathrm{TM}}$. Acetaminophen injection. [package insert]. Mallinckrodt Pharmaceuticals: Webster Groves MO, March 2018.

20. Hakim M, Anderson BJ, Walia H, et al. Acetaminophen pharmacokinetics in severely obese adolescents and young adults. Paediatr Anaesth 2019;29: 20-26.

21. Barshop NJ, Capparelli EV, Sirlin CB, Schwimmer JB, Lavine JE. Acetaminophen pharmacokinetics in children with nonalcoholic fatty liver disease. $J$ Pediatr Gastroenterol Nutr 2011;52: 198-202.

22. Vaughns JD, Ziesenitz VC, Williams EF, et al. Use of fentanyl in adolescents with clinically severe obesity undergoing bariatric surgery: A pilot study. Paediatr Drugs 2017;19: 251-257. 
23. Johnson PN, Skrepnek GH, Golding CL, Owora AH, Thomas AN, Miller JL. Relationship between rate of fentanyl infusion and time to achieve sedation in nonobese and obese critically ill children. Am J Health Syst Pharm 2017;74: 1174-1183.

24. Koshida R, Nakashima E, Taniguchi N, Tsuji A, Benet LZ, Ichimura F. Prediction of the distribution volumes of cefazolin and tobramycin in obese children based on physiological pharmacokinetic concepts. Pharm Res 1989;6: 486-491.

25. ClinicalTrials.gov. Pharmacokinetics of understudied drugs administered to children per standard of care. Identifier NCT 01431326. National Library of Medicine (US): Bethesda MD, 2018.

26. ClinicalTrials.gov. Safety and pharmacokinetics of clindamycin in pediatric subjects with BMI $\geq 85$ th percentile. Identifiers NCT 01744730 and NCT 01728363 . National Library of Medicine (US): Bethesda MD, 2016.

27. Smith MJ, Gonzalez D, Goldman JL, et al. Pharmacokinetics of clindamycin in obese and nonobese children. Antimicrob Agents Chemother 2017;61.

28. Bhave G, Neilson EG. Body fluid dynamics: Back to the future. J Am Soc Nephrol 2011;22: 2166-2181. 
29. Pai MP, Bearden DT. Antimicrobial dosing considerations in obese adult patients. Pharmacotherapy 2007;27: 1081-1091.

30. Choi JJ, Moffett BS, McDade EJ, Palazzi DL. Altered gentamicin serum concentrations in obese pediatric patients. Pediatr Infect Dis J 2011;30: 347-349.

31. Moffett BS, Kam C, Galati M, et al. The "ideal" body weight for pediatric gentamicin dosing in the era of obesity: A population pharmacokinetic analysis. Ther Drug Monit 2018;40: 322-329.

32. Sinha J, Duffull SB, AI-Sallami HS. A review of the methods and associated mathematical models used in the measurement of fat-free mass. Clin Pharmacokinet 2018;57: 781-795.

33. Al-Sallami HS, Goulding A, Grant A, Taylor R, Holford N, Duffull SB. Prediction of fat-free mass in children. Clin Pharmacokinet 2015;54: 1169-1178.

34. Blouin RA, Bauer LA, Miller DD, Record KE, Griffen WO, Jr. Vancomycin pharmacokinetics in normal and morbidly obese subjects. Antimicrob Agents Chemother 1982;21: 575-580. 
35. Vance-Bryan K, Guay DR, Gilliland SS, Rodvold KA, Rotschafer JC. Effect of obesity on vancomycin pharmacokinetic parameters as determined by using a Bayesian forecasting technique. Antimicrob Agents Chemother 1993;37: 436-440.

36. Bauer LA, Black DJ, Lill JS. Vancomycin dosing in morbidly obese patients. Eur J Clin Pharmacol 1998;54: 621-625.

37. Le J, Capparelli EV, Wahid U, et al. Bayesian estimation of vancomycin pharmacokinetics in obese children: Matched case-control study. Clin Ther 2015;37: $1340-1351$.

38. Camaione L, Elliott K, Mitchell-Van Steele A, Lomaestro B, Pai MP. Vancomycin dosing in children and young adults: Back to the drawing board. Pharmacotherapy 2013;33: 1278-1287.

39. Kufel WD, Seabury RW, Mogle BT, Beccari MV, Probst LA, Steele JM. Readiness to implement vancomycin monitoring based on area under the concentration-time curve: A cross-sectional survey of a national health consortium. Am J Health Syst Pharm 2019;76: 889-894. 
40. Nassar L, Hadad S, Gefen A, et al. Prospective evaluation of the dosing regimen of vancomycin in children of different weight categories. Curr Drug Saf 2012;7: 375-381.

41. Eiland LS, Sonawane KB. Vancomycin dosing in healthy-weight, overweight, and obese pediatric patients. J Pediatr Pharmacol Ther 2014;19: 182-188.

42. Heble DE, Jr., McPherson C, Nelson MP, Hunstad DA. Vancomycin trough concentrations in overweight or obese pediatric patients. Pharmacotherapy 2013;33: $1273-1277$.

43. Madigan T, Sieve RM, Graner KK, Banerjee R. The effect of age and weight on vancomycin serum trough concentrations in pediatric patients. Pharmacotherapy 2013;33: 1264-1272.

44. Miller M, Miller JL, Hagemann TM, Harrison D, Chavez-Bueno S, Johnson PN. Vancomycin dosage in overweight and obese children. Am J Health Syst Pharm 2011;68: 2062-2068.

45. Moffett BS, Kim S, Edwards MS. Vancomycin dosing in obese pediatric patients. Clin Pediatr (Phila) 2011;50: 442-446. 
46. Lang JE, Bunnell HT, Hossain MJ, et al. Being overweight or obese and the development of asthma. Pediatrics 2018;142.

47. McGarry ME, Castellanos E, Thakur N, et al. Obesity and bronchodilator response in black and Hispanic children and adolescents with asthma. Chest 2015;147: $1591-1598$.

48. Borrell LN, Nguyen EA, Roth LA, et al. Childhood obesity and asthma control in the GALA II and SAGE II studies. Am J Respir Crit Care Med 2013;187: 697-702.

49. Rastogi D. Quantifying the contribution of obesity to incident childhood asthma: It's about time. Pediatrics 2018;142.

50. Forno E, Lescher R, Strunk R, et al. Decreased response to inhaled steroids in overweight and obese asthmatic children. J Allergy Clin Immunol 2011;127: 741-749.

51. Messinger MM, Moffett BS, Wilfong A. Impact of body habitus on phenytoin levels following fosphenytoin loading dose in pediatric patients. Ther Drug Monit 2015;37: 772-775. 
52. van Rongen A, Vaughns JD, Moorthy GS, Barrett JS, Knibbe CA, van den Anker JN. Population pharmacokinetics of midazolam and its metabolites in overweight and obese adolescents. Br J Clin Pharmacol 2015;80: 1185-1196.

53. Brill MJ, van Rongen A, Houwink AP, et al. Midazolam pharmacokinetics in morbidly obese patients following semi-simultaneous oral and intravenous administration: A comparison with healthy volunteers. Clin Pharmacokinet 2014;53: 931-941.

54. van Rongen A, Brill MJE, Vaughns JD, et al. Higher midazolam clearance in obese adolescents compared with morbidly obese adults. Clin Pharmacokinet 2018;57: 601-611.

55. Monagle P, Chan AKC, Goldenberg NA, et al. Antithrombotic therapy in neonates and children: Antithrombotic therapy and prevention of thrombosis, 9th ed: American College of Chest Physicians evidence-based clinical practice guidelines. Chest 2012;141: e737S-e801S.

56. Matson KL, Horton ER, Capino AC, Advocacy Committee for the Pediatric Pharmacy Advocacy G. Medication dosage in overweight and obese children. J Pediatr Pharmacol Ther 2017;22: 81-83. 
57. Lewis TV, Johnson PN, Nebbia AM, Dunlap M. Increased enoxaparin dosing is required for obese children. Pediatrics 2011;127: e787-790.

58. Mushtaq A, Vaughns JD, Ziesenitz VC, Nadler EP, van den Anker JN. Use of enoxaparin in obese adolescents during bariatric surgery--a pilot study. Obes Surg 2015;25: 1869-1874.

59. Ziesenitz V, Vaughns J, Williams E, Nadler E, Mikus G, van den Anker J. P116 prophylactic use of enoxaparin during bariatric surgery in adolescents with severe obesity. Archives of Disease in Childhood 2019;104: e65.63-e66.

60. Richard AA, Kim S, Moffett BS, Bomgaars L, Mahoney D, Jr., Yee DL. Comparison of anti-Xa levels in obese and non-obese pediatric patients receiving treatment doses of enoxaparin. J Pediatr 2013;162: 293-296.

61. Hoffman S, Braunreiter C. Reduced dosing of enoxaparin for venous thromboembolism in overweight and obese adolescents: A single institution retrospective review. Res Pract Thromb Haemost 2017;1: 188-193.

62. Taylor BN, Bork SJ, Kim S, Moffett BS, Yee DL. Evaluation of weight-based dosing of unfractionated heparin in obese children. J Pediatr 2013;163: 150-153. 
63. Moffett BS, Teruya J, Petit C. Heparin dosing in obese pediatric patients in the cardiac catheterization laboratory. Ann Pharmacother 2011;45: 876-880.

64. Moffett BS, Ung M, Bomgaars L. Risk factors for elevated inr values during warfarin therapy in hospitalized pediatric patients. Pediatr Blood Cancer 2012;58: 941944.

65. Arslanian S, Bacha F, Grey M, Marcus MD, White NH, Zeitler P. Evaluation and management of youth-onset type 2 diabetes: A position statement by the American Diabetes Association. Diabetes Care 2018;41: 2648-2668.

66. American Diabetes Association. 13. Children and adolescents: Standards of medical care in diabetes-2019. Diabetes Care 2019;42: S148-S164.

67. van der Aa MP, Elst MA, van de Garde EM, van Mil EG, Knibbe CA, van der Vorst MM. Long-term treatment with metformin in obese, insulin-resistant adolescents: Results of a randomized double-blinded placebo-controlled trial. Nutr Diabetes 2016;6: e228.

68. van der Aa MP, Hoving V, van de Garde EM, de Boer A, Knibbe CA, van der Vorst MM. The effect of eighteen-month metformin treatment in obese adolescents: 
Comparison of results obtained in daily practice with results from a clinical trial. $J$ Obes 2016;2016: 7852648 .

69. Yanovski JA, Krakoff J, Salaita CG, et al. Effects of metformin on body weight and body composition in obese insulin-resistant children: A randomized clinical trial. Diabetes 2011;60: 477-485.

70. van der Aa MP, Elst MA, van Mil EG, Knibbe CA, van der Vorst MM. Metformin: An efficacy, safety and pharmacokinetic study on the short-term and long-term use in obese children and adolescents - study protocol of a randomized controlled study. Trials 2014;15: 207.

71. van Rongen A, van der Aa MP, Matic M, et al. Increased metformin clearance in overweight and obese adolescents: A pharmacokinetic substudy of a randomized controlled trial. Paediatr Drugs 2018;20: 365-374.

72. Sam WJ, Roza O, Hon YY, et al. Effects of SLC22A1 polymorphisms on metformin-induced reductions in adiposity and metformin pharmacokinetics in obese children with insulin resistance. J Clin Pharmacol 2017;57: 219-229.

73. Glucophage. Metformin hydrochloride extended release tablets [package insert]. Bristol-Myers Squibb Company: Princeton NJ USA, May 2018. 
74. SmPC. Glucophage. Metformin hydrochloride film-coated tablets Merck Serono Ltd: UK, December 2016.

75. Shakhnovich V, Abdel-Rahman S, Friesen CA, et al. Lean body weight dosing avoids excessive systemic exposure to proton pump inhibitors for children with obesity. Pediatr Obes 2019;14.

76. Shakhnovich V, Smith PB, Guptill JT, et al. Obese children require lower doses of pantoprazole than nonobese peers to achieve equal systemic drug exposures. $J$ Pediatr 2018;193: 102-108 e101.

77. Shakhnovich V, Brian Smith P, Guptill JT, et al. A population-based pharmacokinetic model approach to pantoprazole dosing for obese children and adolescents. Paediatr Drugs 2018;20: 483-495.

78. Protonix. Pantoprazole sodium delayed release tablets [package insert]. Wyeth Pharmaceuticals LLC: Philadelphia PA USA, April 2019.

79. Ollivier C, Mulugeta YL, Ruggieri L, Saint-Raymond A, Yao L. Paediatric extrapolation: A necessary paradigm shift. Br J Clin Pharmacol 2019;85: 675-679. 
80. Ameer B, Weintraub MA. Pediatric obesity: Influence on drug dosing and therapeutics. J Clin Pharmacol 2018;58 Suppl 10: S94-S107.

81. Barrett JS, Bishai R, Bucci-Rechtweg C, et al. Challenges and opportunities in the development of medical therapies for pediatric populations and the role of extrapolation. Clin Pharmacol Ther 2018;103: 419-433.

82. Van Driest SL, Choi L. Real-world data for pediatric pharmacometrics: Can we upcycle clinical data for research use? Clin Pharmacol Ther 2019;0.

83. Gonzalez D, Melloni C, Yogev R, et al. Use of opportunistic clinical data and a population pharmacokinetic model to support dosing of clindamycin for premature infants to adolescents. Clin Pharmacol Ther 2014;96: 429-437.

84. Hume M, Lewis LL, Nelson RM. Meeting the goal of concurrent adolescent and adult licensure of HIV prevention and treatment strategies. Journal of Medical Ethics 2017;43: 857-860.

85. National Institutes of Health. Revision: NIH policy and guidelines on the inclusion of individuals across the lifespan as participants in research involving human subjects. National Institutes of Health: Bethesda MD, 2017. 
86. Natale S, Bradley J, Nguyen WH, et al. Pediatric obesity: Pharmacokinetic alterations and effects on antimicrobial dosing. Pharmacotherapy 2017;37: 361-378.

87. Vaughns JD, Ziesenitz VC, van den Anker JN. Clinical pharmacology of frequently used intravenous drugs during bariatric surgery in adolescents. Curr Pharm Des 2015;21: 5650-5659.

88. Rowe S, Siegel D, Benjamin DK, Jr., Best Pharmaceuticals for Children Act Pediatric Trials Network Administrative Core C. Gaps in drug dosing for obese children: A systematic review of commonly prescribed emergency care medications. Clin Ther 2015;37: 1924-1932.

89. Kyler KE, Wagner J, Hosey-Cojocari C, Watt K, Shakhnovich V. Drug dose selection in pediatric obesity: Available information for the most commonly prescribed drugs to children. Paediatr Drugs 2019;21: 357-369.

90. Krzyżaniak N, Pawłowska I, Bajorek B. Review of drug utilization patterns in NICUs worldwide. Journal of Clinical Pharmacy and Therapeutics 2016;41: 612-620.

91. Kosse RC, Koster ES, de Vries TW, Bouvy ML. Drug utilisation among Dutch adolescents: A pharmacy prescription records study. Arch Dis Child 2018. 
92. Chai G, Governale L, McMahon AW, Trinidad JP, Staffa J, Murphy D. Trends of outpatient prescription drug utilization in US children, 2002-2010. Pediatrics 2012;130: 23-31.

93. Kaplan LM, Golden A, Jinnett K, et al. Perceptions of barriers to effective obesity care: Results from the national action study. Obesity (Silver Spring) 2018;26: 61-69.

94. Green DJ, Zineh I, Burckart GJ. Pediatric drug development: Outlook for sciencebased innovation. Clin Pharmacol Ther 2018;103: 376-378. 


\section{Figure 1}

English language PubMed articles from January 1970 through November 2018 were searched using the following search string:

(((obesity[Title/Abstract] OR obese[Title/Abstract] OR overweight[Title/Abstract] AND (pharmacokinetics[Title/Abstract] OR pharmacodynamics[Title/Abstract] OR PK[Title/Abstract] OR PD[Title/Abstract] OR PK/PD[Title/Abstract] OR (medication[Title/Abstract] OR dosing[Title/Abstract])) AND ("infant"[MeSH Terms] OR "child"[MeSH Terms] OR "adolescent"[MeSH Terms])) OR (("pharmacokinetics"[Subheading] OR "pharmacokinetics"[All Fields] OR "pharmacokinetics"[MeSH Terms]) AND ("obesity"[MeSH Terms] OR "obesity"[All Fields]) AND ("infant”[MeSH Terms] OR ("infant"[MeSH Terms] OR "child"[MeSH Terms] OR "adolescent"[MeSH Terms])))) AND ("drug name"[MeSH Terms] OR "drug name"[All Fields])

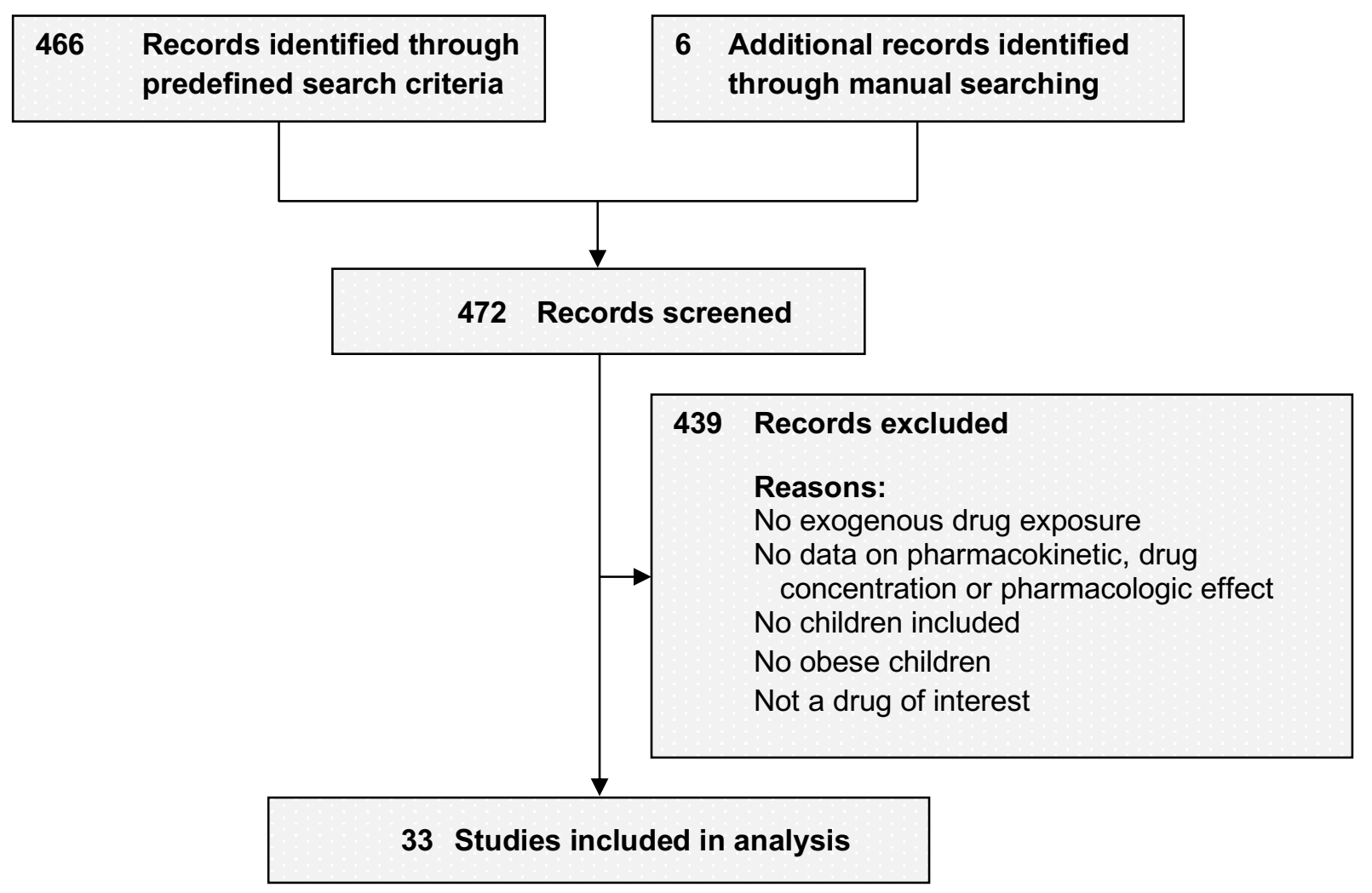


Table 1. Presence of Published Pediatric Obesity Studies Among 70 Commonly Used Medications in Children and Adolescents ${ }^{a}$

Therapeutic Category

Drug Class

Drug Name

Study of

Pediatric

Obesity $^{b}$

ANALGESIC:

Non-Opiate

acetaminophen

ibuprofen

ketorolac

Opiate

aspirin

fentanyl

morphine

hydromorphone

methadone

oxycodone

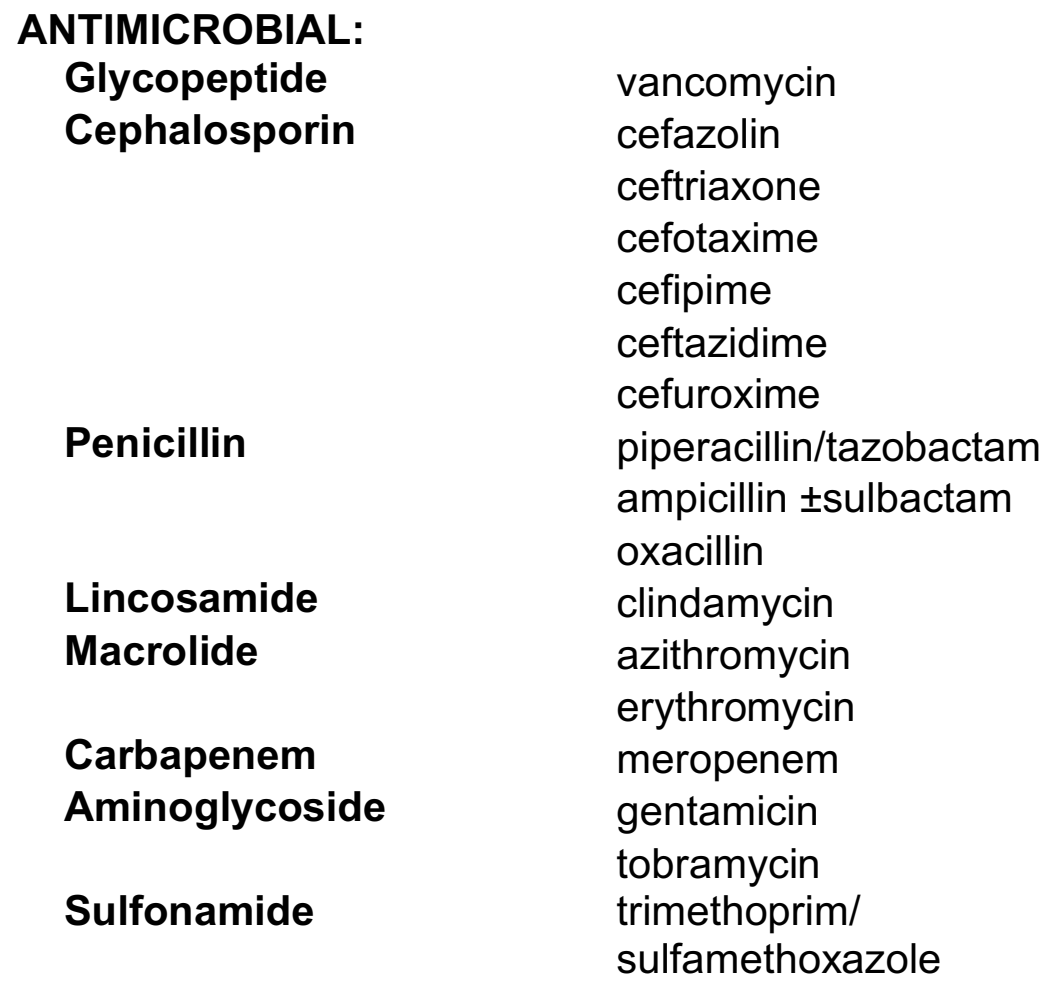

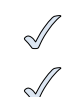


ASTHMA, ALLERGY \&

INFLAMMATION:

Glucocorticoid dexamethasone

methylprednisolone

hydrocortisone

budesonide

prednisone

Antihistamine

diphenhydramine

cetirizine

Beta-Agonist

albuterol

CARDIOVASCULAR:

Diuretic

furosemide

chlorothiazide

spironolactone

ACE Inhibitor

bumetanide

enalapril

captopril

Calcium Channel Blocker nicardipine

amlodipine

Beta Blocker

metoprolol

propranolol

labetalol

\section{CENTRAL NERVOUS SYSTEM:}

Anti-epileptic

Sedative lorazepam

levetiracetam

diazepam

phenobarbital

fosphenytoin or

phenytoin

clonazepam

topiramate

gabapentin

oxcarbazepine

clonidine

midazolam 
COAGULATION:

Anticoagulant

heparin

enoxaparin

warfarin

Antifibrinolytic

aminocaproic acid

tranexamic acid

\section{DIABETES:}

Biguanide

metformin

Hormone

insulin

GASTROINTESTINAL:

H2 Antagonist

Proton Pump Inhibitor

Motility

5HT3 Antagonist ranitidine

famotidine

pantoprazole

omeprazole

esomeprazole

metoclopramide

aBased on drug exposures, accumulated from 42 United States pediatric intensive care units, for patients ranging in age from 1 through 17 years. See text for details.

${ }^{\mathrm{b}}$ Checkmark indicates one or more published reports. 